\title{
Breakfast replacement with a liquid formula improves glycaemic variability in patients with type 2 diabetes: a randomised clinical trial
}

\author{
Jiahui Peng†, Jingyi Lu†, Xiaojing Ma*, Lingwen Ying, Wei Lu, Wei Zhu, Yuqian Bao and Jian Zhou* \\ Shanghai Key Laboratory of Diabetes Mellitus, Department of Endocrinology and Metabolism, Shanghai Jiao Tong University \\ Affiliated Sixth People's Hospital, Shanghai Clinical Center for Diabetes, Shanghai Diabetes Institute, Shanghai, People's \\ Republic of China \\ (Submitted 19 September 2018 - Final revision received 7 November 2018 - Accepted 18 November 2018 - First published online 16 January 2019)
}

\section{Abstract}

There is emerging evidence that glycaemic variability (GV) plays an important role in the development of diabetic complications. The current study aimed to compare the effects of lifestyle intervention (LI) with and without partial meal replacement (MR) on GV. A total of 123 patients with newly diagnosed and untreated type 2 diabetes (T2D) were randomised to receive either LI together with breakfast replacement with a liquid formula (LI + MR) ( $n$ 62) or LI alone ( $n$ 61) for 4 weeks and completed the study. Each participant was instructed to have three main meals per $\mathrm{d}$ and underwent 72 -h continuous glucose monitoring (CGM) both before and after intervention. Measures of GV assessed by CGM included the incremental AUC of postprandial blood glucose (AUCpp), standard deviation of blood glucose (SDBG), glucose CV and mean amplitude of glycaemic excursions (MAGE). After a 4-week intervention, the improvements in systolic blood pressure ( $P=0 \cdot 046)$ and time in range $(P=0.033)$ were more pronounced in the LI + MR group than in the LI group. Furthermore, LI + MR caused significantly greater improvements in all GV metrics including SDBG $(P=0 \cdot 005)$, CV $(P=0 \cdot 002)$, MAGE $(P=0 \cdot 016)$ and AUCpp $(P<0 \cdot 001)$ than did LI. LI + MR ( $v$. LI) was independently associated with improvements in GV after adjustment of covariates (all $P<0 \cdot 05$ ). Our study showed that LI + MR led to significantly greater improvements in GV compared with LI, suggesting that LI + MR could be an effective treatment to alleviate glucose excursions.

Key words: Meal replacement: Lifestyle: Glycaemic variability: Type 2 diabetes

It is well established that nutrition plays an important role in the management and prevention of type 2 diabetes (T2D) through its effect on weight and metabolic control. Therefore, the American Diabetes Association has recommended that nutrition therapy should be regarded as an effective component of the overall treatment plan for all patients with either type 1 diabetes or $\mathrm{T} 2 \mathrm{D}^{(1)}$. One of the major obstacles in the implementation of nutrition therapy is poor compliance, which may be improved using a meal replacement (MR) programme. In patients with T2D, the MR has been demonstrated to exert beneficial effects on weight management ${ }^{(2-6)}$, lipids ${ }^{(3,4,6)}$, blood pressure $(\mathrm{BP})^{(3)}$, liver function ${ }^{(3)}$, insulin sensitivity ${ }^{(3,4,7,8)}$ and glycaemic control $^{(2-9)}$.

The sustained chronic hyperglycaemia, hypoglycaemic episodes and glycaemic variability (GV) have been depicted as the three main components of dysglycaemia in diabetes ${ }^{(10)}$. Of them, GV refers to short-term (intra-day) or long-term (inter-day) fluctuations in glucose around the mean value ${ }^{(11)}$. Although chronic hyperglycaemia is the main risk factor for diabetic complications ${ }^{(12-14)}$, there is emerging evidence that GV contributes independently to the development of diabetic complications in both type 1 diabetes $^{(15,16)}$ and T2D ${ }^{(17,18)}$. In vivo ${ }^{(19)}$, in vitro ${ }^{(20)}$ and clinical $^{(21,22)}$ studies demonstrated that glucose fluctuations induce endothelial dysfunction and oxidative stress, a key player in the pathogenesis of diabetic complications ${ }^{(23)}$. Previous studies on MR in patients with T2D mainly focused on indices of hyperglycaemia including fasting and postprandial glucose and, most importantly, glycated $\mathrm{Hb}$ $\left(\mathrm{HbA}_{1 \mathrm{c}}\right)^{(2,4-6,9)}$, which reflects the average glucose level during the past 2-3 months. However, none has examined whether MR exerts beneficial effects on GV. Therefore, we conducted this randomised clinical trial (NCT02248714) to evaluate the effects of lifestyle intervention (LI) with or without breakfast replacement with a liquid formula on the indices of $\mathrm{GV}$, as assessed by continuous glucose monitoring (CGM) and markers of metabolic control.

\footnotetext{
Abbreviations: AUCpp, AUC of postprandial blood glucose; BP, blood pressure; CGM, continuous glucose monitoring; FPG, fasting plasma glucose; GA, glycated albumin; GLP-1, glucagon-like peptide 1; GV, glycaemic variability; $\mathrm{HbA}_{1 \mathrm{c}}$ glycated $\mathrm{Hb}$; LI, lifestyle intervention; MAGE, mean amplitude of glycaemic excursions; MR, meal replacement; OGTT, oral glucose tolerance test; SDBG, standard deviation of blood glucose; T2D, type 2 diabetes; TIR, time in range.
}

* Corresponding authors: X. Ma, fax +8621 64368031, email maxiaojing@sjtu.edu.cn; J. Zhou, fax +86 2164368031 , email zhoujian@sjtu.edu.cn

$\dagger$ These two authors contributed equally to this work. 


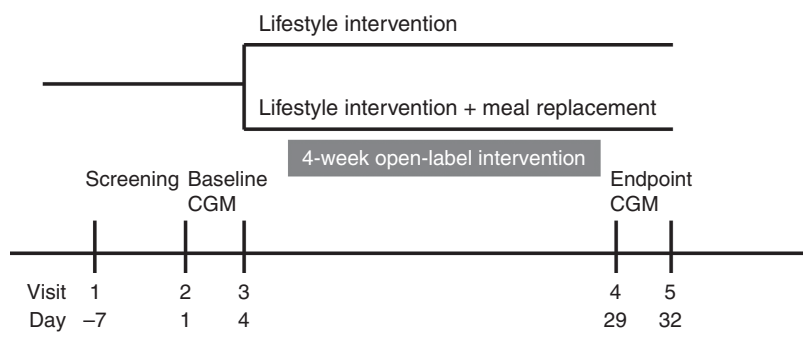

Fig. 1. Schematic outline of study conduction. CGM, continuous glucose monitoring.

\section{Methods \\ Study populations}

In this study, subjects referred to the outpatient clinic at the Department of Endocrinology of Shanghai Jiao Tong University Affiliated Sixth People's Hospital were enroled from March 2011 to March 2018. The inclusion criteria were as follows: (1) newly diagnosed and untreated T2D, (2) BMI $\geq 18.5 \mathrm{~kg} / \mathrm{m}^{2}$ and (3) $\mathrm{HbA}_{1 \mathrm{c}} \geq 6.5 \%$. The exclusion criteria were as follows: (1) severe liver disease, (2) severe kidney disease, (3) history of unstable angina or myocardial infarction or New York Heart Association class III or IV congestive heart failure, (4) acute or chronic disorders interfering with digestion or absorption, (4) history of malignancy or mental disorders and (5) pregnancy or lactation. T2D was defined in accordance with the 1999 WHO criteria.

Following study enrolment (visit 1), each subject underwent a 75-g oral glucose tolerance test (OGTT). Participants who met the eligibility criteria were then monitored continuously for $72 \mathrm{~h}$ (visit 2 to visit 3) with a retrospective CGM system (Medtronics). After $3 \mathrm{~d}$ of CGM (visit 3), the subjects were randomised (1:1) to receive either $\mathrm{LI}$ or $\mathrm{LI}+\mathrm{MR}$ for 4 weeks. At the end of the 4-week intervention, subjects underwent another CGM from visit 4 (day 29) to visit 5 (day 32), at which a final OGTT was conducted in the fasting state. The schematic outline of study conduction is illustrated in Fig. 1.

The study protocol was in accordance with the Helsinki Declaration and approved by the Ethics Committees of Shanghai Jiao Tong University Affiliated Sixth People's Hospital. Written informed consent was obtained from all participants.

This trial is registered at www.ClinicalTrials.gov with clinical trial registration number NCT02248714.

\section{Intervention}

Before the intervention (visit 3), both groups received lifestyle education delivered by an experienced nutritionist who assessed each patient's medical history and clinical characteristics and prescribed an individualised meal plan comprising the quantity and type of food products, ensuring a total daily energy intake of $105-126 \mathrm{~kJ} / \mathrm{kg}$ ideal body weight $/ \mathrm{d}$. Participants were instructed to have three main meals (i.e. breakfast, lunch and dinner) per $\mathrm{d}$ and avoid snacks. The prescribed diet consisted of $45-60 \%$ of energy from carbohydrate, $25-35 \%$ from fat and $15-20 \%$ from protein, according to the Chinese guidelines to the medical nutrition therapy for
Table 1. The composition of Glucerna SR powder

\begin{tabular}{|c|c|c|}
\hline Nutrient & Per $100 \mathrm{~g}$ & One serving $(52 \mathrm{~g})$ \\
\hline Energy (kJ) & $1774 \cdot 8$ & $925 \cdot 1$ \\
\hline Protein (g) & $21 \cdot 2$ & $11 \cdot 0$ \\
\hline Fat $(g)$ & $15 \cdot 4$ & 8.0 \\
\hline Carbohydrate $(\mathrm{g})$ & 55.9 & $29 \cdot 1$ \\
\hline Total dietary fibre (g) & 3.5 & 1.8 \\
\hline Carnitine (mg) & $96 \cdot 2$ & $50 \cdot 0$ \\
\hline Taurine (mg) & 38.5 & $20 \cdot 0$ \\
\hline Inositol (mg) & $384 \cdot 6$ & $200 \cdot 0$ \\
\hline \multicolumn{3}{|l|}{ Vitamins } \\
\hline Vitamin A (mg) & 1.0 & 0.5 \\
\hline Vitamin A (palmitate) (mg) & $1 \cdot 2$ & 0.6 \\
\hline Vitamin $A$ ( $\beta$-carotene) (mg) & 0.8 & 0.4 \\
\hline Vitamin $D_{3}(\mu \mathrm{g})$ & $4 \cdot 8$ & 2.5 \\
\hline Vitamin E (mg) & $40 \cdot 0$ & $20 \cdot 7$ \\
\hline Vitamin $\mathrm{K}_{1}(\mu \mathrm{g})$ & 38.5 & $20 \cdot 0$ \\
\hline Vitamin C (mg) & $115 \cdot 4$ & $60 \cdot 0$ \\
\hline Folic acid $(\mu \mathrm{g})$ & 385 & 200 \\
\hline Vitamin $B_{1}(\mu \mathrm{g})$ & 731 & 380 \\
\hline Vitamin $B_{2}(\mu \mathrm{g})$ & 827 & 430 \\
\hline Vitamin $\mathrm{B}_{6}(\mathrm{mg})$ & 1.7 & 0.9 \\
\hline Vitamin $B_{12}(\mu \mathrm{g})$ & $5 \cdot 8$ & 3.0 \\
\hline Niacin (mg) & 9.6 & $5 \cdot 0$ \\
\hline Pantothenic acid (mg) & 4.8 & 2.5 \\
\hline Biotin $(\mu \mathrm{g})$ & $144 \cdot 2$ & $75 \cdot 0$ \\
\hline Choline (mg) & $192 \cdot 3$ & $100 \cdot 0$ \\
\hline \multicolumn{3}{|l|}{ Minerals } \\
\hline $\mathrm{Na}(\mathrm{mg})$ & 404 & 210 \\
\hline $\mathrm{K}(\mathrm{mg})$ & 712 & 370 \\
\hline Chloride (mg) & 681 & 354 \\
\hline $\mathrm{Ca}(\mathrm{mg})$ & 481 & 250 \\
\hline$P(m g)$ & 452 & 235 \\
\hline $\mathrm{Mg}(\mathrm{mg})$ & $192 \cdot 3$ & $100 \cdot 0$ \\
\hline $\mathrm{Fe}(\mathrm{mg})$ & $8 \cdot 7$ & 4.5 \\
\hline $\mathrm{Zn}(\mathrm{mg})$ & $7 \cdot 4$ & 3.8 \\
\hline $\mathrm{Mn}(\mathrm{mg})$ & 1.9 & 1.0 \\
\hline $\mathrm{Cu}(\mu \mathrm{g})$ & 962 & 500 \\
\hline $\mathrm{Se}(\mu \mathrm{g})$ & 34.6 & $18 \cdot 0$ \\
\hline $\mathrm{Cr}(\mathrm{mg})$ & 0.2 & 0.1 \\
\hline
\end{tabular}

diabetes. Breakfast, lunch and dinner comprised approximately 20, 40 and $40 \%$ of total daily energy intake, respectively. Participants were also advised to replace refined carbohydrates with low-glycaemic index foods such as whole grains and vegetables, avoid sugar-sweetened beverages, minimise the consumption of foods with added sugar, limit the intake of fried and fatty foods and cut down on salt and cooking oil. Besides, they were encouraged to engage in $150 \mathrm{~min}$ or more of moderate-intensity physical activity per week and reduce the amount of time spent being sedentary. At the end of week 2, all study subjects were asked to visit the nutritionist again and the instructions on diet and exercise were reinforced.

In addition to the lifestyle education, participants in the LI+ MR group were instructed to replace breakfast with Glucerna SR (Abbot Nutrition) powder $52 \mathrm{~g}$ dissolved in $200 \mathrm{ml}$ of warm water, providing $923.0 \mathrm{~kJ}, 29.1 \mathrm{~g}$ of carbohydrate, $11.0 \mathrm{~g}$ of protein and $8.0 \mathrm{~g}$ fat. The composition of Glucerna SR is listed in Table 1 . The average energy content of the breakfast in the LI group was $1450.8 \mathrm{~kJ}$, containing $42.6 \mathrm{~g}$ of carbohydrate, $11.8 \mathrm{~g}$ of fat and $18.2 \mathrm{~g}$ of protein. The macronutrients composition and energy content in lunch and dinner did not differ between the two groups. 


\section{Continuous glucose monitoring parameters}

Time in range (TIR) was defined as the percentage of time in the target glucose range of $3.9-10.0 \mathrm{mmol} / \mathrm{l}$ during a $24-\mathrm{h}$ period. The incremental AUC of postprandial blood glucose (AUCpp) during CGM was calculated as the area between the glucose concentration-time curve and the pre-prandial baseline glucose value measured at $4 \mathrm{~h}$ after each meal. Intra-day $\mathrm{GV}$ parameters include the standard deviation of blood glucose (SDBG) values, glucose CV and the mean amplitude of glycaemic excursions (MAGE). CV was calculated by dividing the SD by the mean of the corresponding glucose readings. MAGE was calculated as the arithmetic mean of the differences between consecutive peaks and nadirs. Only excursions of more than 1SD of the mean glycaemic values were considered for the calculation of MAGE.

\section{Anthropometric and biochemical measurements}

Anthropometric and biochemical parameters were measured at visit 1 (baseline) and visit 4 (post-intervention). BMI was calculated as the weight ( $\mathrm{kg}$ ) divided by squared height ( $\mathrm{m})$. BP was measured twice using a standard mercury sphygmomanometer and the average was documented. Venous blood samples were collected after an overnight fast. Biochemical parameters including fasting plasma glucose (FPG), 2-h plasma glucose (2h-PG) during OGTT, $\mathrm{HbA}_{1 \mathrm{c}}$, glycated albumin (GA), insulin, TAG, total cholesterol, HDL-cholesterol and LDL-cholesterol were assayed as previously reported $^{(24)}$.

Insulin sensitivity was evaluated by homoeostasis model assessments of insulin resistance (HOMA-IR) and Matsuda index using the following formulae: HOMA-IR = fasting insulin (in $\mathrm{mU} / \mathrm{l}$ ) $\times$ FPG (in $\mathrm{mmol} / \mathrm{l}) / 22.5$ and Matsuda index $=10000$ / square root of $(\mathrm{FPG}$ (in $\mathrm{mmol} / \mathrm{l}) \times$ fasting insulin (in $\mathrm{mU} / \mathrm{l})) \times($ mean glucose $\times$ mean insulin during OGTT) ${ }^{(25)}$. Early phase $(0-30 \mathrm{~min})$ and total (0-120 $\mathrm{min})$ incremental insulin response to the increase in plasma glucose concentration $(\Delta \mathrm{I} / \Delta \mathrm{G})$ were calculated as indices of insulin secretion.

\section{Determination of sample size and statistical analyses}

Because the effect of MR on GV has not been reported previously, sample size calculation was not performed and a convenience sample size was adopted. Based on the current sample size, our study had more than $80 \%$ power $(\alpha=0.05)$ in each arm to detect the effect sizes of $150 \cdot 0$ (sD 368.0) $\mathrm{min} \times$ $\mathrm{mmol} / \mathrm{l}, 0.3(\mathrm{sD} 0 \cdot 7$ ) $\mathrm{mmol} / \mathrm{l}, 3.0$ (sD 8.0)\% and 0.8 (sD 2.1) mmol/1 for AUCpp, SDBG, CV and MAGE, respectively.

The primary outcomes were the improvements in metrics of GV. The secondary outcomes were the improvements in cardiometabolic risk factors including body weight, BMI, BP, lipid profiles and indices of glucose control, insulin secretion and insulin sensitivity.

Continuous variables at baseline were compared between the two groups by Student's $t$ test. Comparison of categorical variables between groups was performed by $\chi^{2}$ tests. Differences in the parameters before and after treatment were analysed by paired $t$ test. The changes $(\Delta)$ in variables were compared with
Student's $t$ test or ANCOVA where indicated. Multivariate linear regression analysis was conducted to examine the risk factors of improvements in metrics of GV after adjusting for covariates. Statistical analyses were performed using SPSS software version 17.0 (SPSS Inc.). A $P$ value of $<0 \cdot 05$ (two tailed) was considered statistically significant.

\section{Results}

In total, 131 patients with T2D met the enrolment criteria and were randomised into the two groups (LI + MR: $n 66$ and LI: $n$ 65). A total of four patients were excluded from the LI $+M R$ group because they were lost to follow up $(n 2)$ or exhibited a major protocol deviation ( $n$ 2). Another four patients were excluded from the LI group because they were lost to follow up. Finally, $62(93.9 \%)$ and 61 (93.8\%) participants completed the study in the LI + MR group and LI group, respectively.

The characteristics of the participants who completed the study before and after the intervention are depicted in Table 2 . At baseline, there were no significant differences in all the clinical parameters except $\Delta \mathrm{I}_{30} / \Delta \mathrm{G}_{30}(\mathrm{LI}+\mathrm{MR}$ : $4.6(\mathrm{sD} 2 \cdot 7) \mathrm{mU} /$ mmol, LI: 6.5 (sD 6.4$) \mathrm{mU} / \mathrm{mmol}, P=0.031$ ). After the 4-week intervention, body weight, BMI, TAG, FPG, 2h-PG, $\mathrm{HbA}_{1 \mathrm{c}}$, GA, TIR, $\Delta \mathrm{I}_{120} / \Delta \mathrm{G}_{120}$, HOMA-IR and Matsuda index were significantly improved in both the LI + MR and LI groups. Compared with baseline, systolic BP $(P<0.001)$ and diastolic BP $(P=0.002)$ at the end of the study were significantly reduced, and $\Delta \mathrm{I}_{30} / \Delta \mathrm{G}_{30}(P=0.013)$ was significantly increased in the $\mathrm{LI}+\mathrm{MR}$ group but not in the LI group. The changes in clinical parameters following interventions did not differ significantly between the two groups, except that the improvement in systolic BP $(P=0.046)$ and TIR $(P=0.033)$ was significantly higher in the LI + MR group compared with the LI group.

In all, 4-week treatment of LI + MR exerted beneficial effects on all the GV-related measures of CGM including SDBG (before: 1.9 (sD 0.7) mmol/1, after: $1.3(\mathrm{sD} 0.5) \mathrm{mmol} / 1, P<0.001$ ), CV (before: 21.5 (sD 7.3) \%, after: 16.8 (sD 6.0) \%, $P<0.001$ ), MAGE (before: $5 \cdot 0$ (sD 2.0) $\mathrm{mmol} / \mathrm{l}$, after: 3.5 (sD 1.5 ) $\mathrm{mmol} / \mathrm{l}$, $P<0.001$ ) and AUCpp (before: 522.5 (sD 335.9) $\mathrm{min} \times \mathrm{mmol} / \mathrm{l}$, after: 234.6 (sD 186.4) $\mathrm{min} \times \mathrm{mmol} / \mathrm{l}, P<0.001)$. In contrast, only MAGE (before: 4.7 (sD 1.7) $\mathrm{mmol} / \mathrm{l}$, after: 4.1 (SD 1.6) $\mathrm{mmol} / \mathrm{l}$, $P=0.032$ ) was significantly reduced in the LI group. LI $+M R$ caused significantly greater improvements in SDBG (LI+MR: $\Delta=-0.6$ (sD 0.8) $\mathrm{mmol} / \mathrm{l}$, LI: $\Delta=-0.2$ (sD 0.7) $\mathrm{mmol} / \mathrm{l}, P=0.005)$, CV (LI +MR: $\Delta=-4 \cdot 7 \quad(\mathrm{sD} \quad 8 \cdot 1) \%, \quad$ LI: $\quad \Delta=-0 \cdot 2 \quad$ (sD $7 \cdot 6) \%$, $P=0 \cdot 002)$, MAGE (LI + MR: $\Delta=-1 \cdot 6($ sD $2 \cdot 3) \mathrm{mmol} / \mathrm{l}$, LI: $\Delta=-0 \cdot 6$ (sD $2 \cdot 1) \mathrm{mmol} / \mathrm{l} \mathrm{mmHg}, P=0 \cdot 016$ ) and AUCpp (LI+MR: $\Delta=$ $-287.8(\mathrm{sD} 368 \cdot 7) \mathrm{min} \times \mathrm{mmol} / \mathrm{l}$, LI: $\Delta=-31 \cdot 2(\mathrm{sD} 301 \cdot 6) \mathrm{min} \times$ $\mathrm{mmol} / \mathrm{l}, P<0.001$ ) compared with LI (Figs 2 and 3).

After combining the two groups of participants, multivariate linear regression analysis revealed that $L I+M R$ and baseline $\Delta \mathrm{I}_{30} / \Delta \mathrm{G}_{30}$ were consistently and independently associated with the improvements in SDBG, CV, MAGE and AUCpp after adjusting for baseline clinical variables including age, sex, BMI, FPG, 2h-PG, $\mathrm{HbA}_{1 \mathrm{c}}, \Delta \mathrm{I}_{120} / \Delta \mathrm{G}_{120}$, Matsuda index and corresponding baseline CGM metrics (i.e. baseline SDBG, CV, MAGE or AUCpp) (Table 3). 
Table 2. Participant characteristics before and after intervention

(Mean values and standard deviations)

\begin{tabular}{|c|c|c|c|c|c|c|c|c|c|c|c|c|}
\hline \multirow[b]{3}{*}{ Parameters } & \multicolumn{6}{|c|}{$\mathrm{LI}+\mathrm{MR}(n$ 62) } & \multicolumn{6}{|c|}{ LI ( $n$ 61) } \\
\hline & \multicolumn{2}{|c|}{ Before } & \multicolumn{2}{|c|}{ After } & \multicolumn{2}{|c|}{ Change } & \multicolumn{2}{|c|}{ Before } & \multicolumn{2}{|c|}{ After } & \multicolumn{2}{|c|}{ Change } \\
\hline & Mean & SD & Mean & SD & Mean & SD & Mean & SD & Mean & SD & Mean & SD \\
\hline Age (years) & $56 \cdot 9$ & $9 \cdot 1$ & \multicolumn{2}{|c|}{-} & \multicolumn{2}{|c|}{-} & 56.5 & 8.4 & \multicolumn{2}{|c|}{-} & \multicolumn{2}{|c|}{ - } \\
\hline Male-female $(n)$ & \multicolumn{2}{|c|}{$30 / 32$} & \multicolumn{2}{|c|}{ - } & \multicolumn{2}{|c|}{ - } & \multicolumn{2}{|c|}{$36 / 25$} & \multicolumn{2}{|c|}{ - } & \multicolumn{2}{|c|}{ - } \\
\hline Body weight (kg) & $67 \cdot 2$ & $10 \cdot 4$ & $65.8+$ & $10 \cdot 2$ & $-1 \cdot 4$ & $1 \cdot 3$ & $70 \cdot 5$ & $10 \cdot 6$ & $69 \cdot 2 \dagger$ & $10 \cdot 7$ & $-1 \cdot 3$ & 1.4 \\
\hline $\operatorname{BMI}\left(\mathrm{kg} / \mathrm{m}^{2}\right)$ & 24.6 & 3.0 & $24 \cdot 1 \dagger$ & 3.0 & -0.5 & 0.5 & $25 \cdot 3$ & $2 \cdot 9$ & $24.8 \dagger$ & $2 \cdot 8$ & -0.5 & 0.5 \\
\hline Systolic BP (mmHg) & $131 \cdot 7$ & $14 \cdot 8$ & $124 \cdot 3 \dagger$ & $13 \cdot 0$ & $-7 \cdot 5^{\star}$ & $11 \cdot 8$ & $128 \cdot 9$ & $12 \cdot 1$ & $126 \cdot 2$ & $14 \cdot 1$ & $-2 \cdot 8$ & $14 \cdot 0$ \\
\hline Diastolic BP $(\mathrm{mmHg})$ & 80.4 & $9 \cdot 2$ & $76 \cdot 1 \dagger$ & 9.3 & $-4 \cdot 4$ & $10 \cdot 8$ & $79 \cdot 7$ & $9 \cdot 0$ & 78.7 & $10 \cdot 1$ & $-1 \cdot 1$ & $8 \cdot 8$ \\
\hline $\mathrm{TC}(\mathrm{mmol} / \mathrm{l})$ & $5 \cdot 1$ & 0.8 & $5 \cdot 0$ & 0.9 & -0.1 & 0.7 & $5 \cdot 1$ & 1.0 & $5 \cdot 0$ & 0.9 & -0.2 & 0.7 \\
\hline TAG $(\mathrm{mmol} / \mathrm{l})$ & 1.9 & $1 \cdot 6$ & $1.5 \dagger$ & 0.8 & -0.4 & $1 \cdot 3$ & 1.6 & 0.6 & $1.4 \dagger$ & 0.5 & -0.2 & 0.5 \\
\hline HDL-cholesterol (mmol/l) & $1 \cdot 2$ & 0.3 & $1 \cdot 2$ & 0.3 & 0.0 & 0.1 & $1 \cdot 2$ & $0 \cdot 2$ & $1 \cdot 2$ & 0.2 & 0.0 & 0.1 \\
\hline LDL-cholesterol (mmol/l) & $3 \cdot 2$ & 0.8 & $3 \cdot 1$ & 0.7 & -0.1 & 0.6 & $3 \cdot 2$ & 0.8 & $3 \cdot 1$ & 0.8 & -0.1 & 0.6 \\
\hline FPG (mmol/l) & $7 \cdot 4$ & 0.8 & $6 \cdot 7 \dagger$ & 0.9 & -0.7 & 0.9 & $7 \cdot 4$ & $1 \cdot 2$ & $6 \cdot 8 \dagger$ & $1 \cdot 1$ & -0.6 & $1 \cdot 0$ \\
\hline 2h-PG (mmol/l) & $15 \cdot 0$ & $2 \cdot 7$ & $12 \cdot 1 \dagger$ & $3 \cdot 2$ & $-2 \cdot 9$ & 3.3 & $15 \cdot 0$ & $2 \cdot 6$ & $13.0 \dagger$ & 3.4 & $-2 \cdot 0$ & $3 \cdot 1$ \\
\hline $\mathrm{HbA}_{1 \mathrm{c}}(\mathrm{mmol} / \mathrm{l})$ & $7 \cdot 2$ & 0.6 & $6 \cdot 7 \dagger$ & 0.4 & -0.5 & 0.4 & $7 \cdot 2$ & 0.6 & $6 \cdot 7 \dagger$ & 0.5 & -0.5 & 0.4 \\
\hline GA $(\%)$ & $18 \cdot 1$ & $2 \cdot 8$ & $15 \cdot 8 \dagger$ & 1.8 & $-2 \cdot 3$ & $2 \cdot 6$ & $17 \cdot 7$ & 2.9 & $15 \cdot 9 \dagger$ & $2 \cdot 4$ & $-1 \cdot 8$ & $2 \cdot 9$ \\
\hline $\operatorname{TIR}(\%)$ & $75 \cdot 7$ & $18 \cdot 4$ & $89.5+$ & $13 \cdot 1$ & $13 \cdot 8^{*}$ & $16 \cdot 6$ & 77.5 & $17 \cdot 8$ & $84.8 \dagger$ & $15 \cdot 2$ & $7 \cdot 3$ & $16 \cdot 5$ \\
\hline$\Delta \mathrm{I}_{30} / \Delta \mathrm{G}_{30}(\mathrm{mU} / \mathrm{mmol})$ & $4 \cdot 6^{*}$ & $2 \cdot 7$ & $5.9 \dagger$ & 3.4 & $1 \cdot 3$ & 3.5 & 6.5 & $6 \cdot 4$ & $6 \cdot 2$ & $6 \cdot 0$ & -0.3 & $5 \cdot 0$ \\
\hline$\Delta \mathrm{l}_{120} / \Delta \mathrm{G}_{120}(\mathrm{mU} / \mathrm{mmol})$ & $10 \cdot 9$ & $9 \cdot 4$ & $15 \cdot 6 \dagger$ & $14 \cdot 3$ & $4 \cdot 7$ & $14 \cdot 6$ & $10 \cdot 7$ & $7 \cdot 3$ & $13 \cdot 2 \dagger$ & $10 \cdot 6$ & -2.5 & 8.9 \\
\hline HOMA-IR & $4 \cdot 2$ & $2 \cdot 9$ & $2.8 \dagger$ & $1 \cdot 7$ & $-1 \cdot 3$ & $2 \cdot 2$ & $4 \cdot 3$ & $2 \cdot 4$ & $3.3 \dagger$ & $2 \cdot 3$ & -1.0 & 1.9 \\
\hline Matsuda index & 3.5 & $2 \cdot 3$ & $4.6 \dagger$ & $3 \cdot 2$ & $1 \cdot 1$ & 1.9 & $3 \cdot 3$ & $2 \cdot 2$ & $4.6 \dagger$ & $3 \cdot 7$ & 1.4 & $2 \cdot 0$ \\
\hline
\end{tabular}

$\mathrm{LI}$, lifestyle intervention; MR, meal replacement; BP, blood pressure; TC, total cholesterol; FPG, fasting plasma glucose; 2h-PG, 2-h plasma glucose during oral glucose tolerance test; $\mathrm{HbA}_{1 \mathrm{c}}$, glycated $\mathrm{Hb} \mathrm{A} \mathrm{A}_{1 \mathrm{c}}$; GA, glycated albumin; TIR, time in range; HOMA-IR, homoeostasis model assessment for insulin resistance.

* $P<0.05$ compared with the LI group. $\dagger P<0.05$ compared with baseline.

\section{Discussion}

Previous studies investigating the effects of full or partial MR on blood glucose have produced conflicting results regarding FPG. However, all these studies reported that MR significantly reduced postprandial glucose levels ${ }^{(3,6,7,9)}$. Postprandial glucose is considered as a contributor to glucose excursions, and there is mounting evidence that postprandial glucose is more strongly associated with CVD than with FPG $^{(26,27)}$. Nevertheless, no study has examined the effect of nutrition therapy on parameters reflecting intra-day GV assessed by CGM. In this study, we found that 4-week replacement of breakfast with Glucerna SR significantly reduced AUCpp, which is in accordance with the findings of previous reports. More importantly, there were significant improvements in GV metrics assessed by CGM including SDBG, CV and MAGE following intervention with LI + MR but not with LI alone. SDBG was reported to be significantly associated with diabetic retinopathy in patients with type 1 diabetes and T2D ${ }^{(28)}$, and Soupal et al. ${ }^{(16)}$ found a positive relationship between SDBG and peripheral neuropathy in type 1 diabetes. Matsutani et $a l^{(17)}$ previously observed in type 1 diabetes that $\mathrm{CV}$ was significantly related to baroreflex sensitivity, an early indicator of cardiovascular autonomic neuropathy. Regarding MAGE, it has been shown to be associated with the presence and severity of coronary artery disease in T2D patients with chest pain ${ }^{(18)}$ and was observed to be predictive of 1 -year major adverse cardiac events in T2D patients with acute myocardial infarction ${ }^{(29)}$.

Regarding the mechanism underlying the impact of MR on intra-day measures of GV (i.e. SDBG, CV and MAGE), since postprandial glucose is an important component of glucose
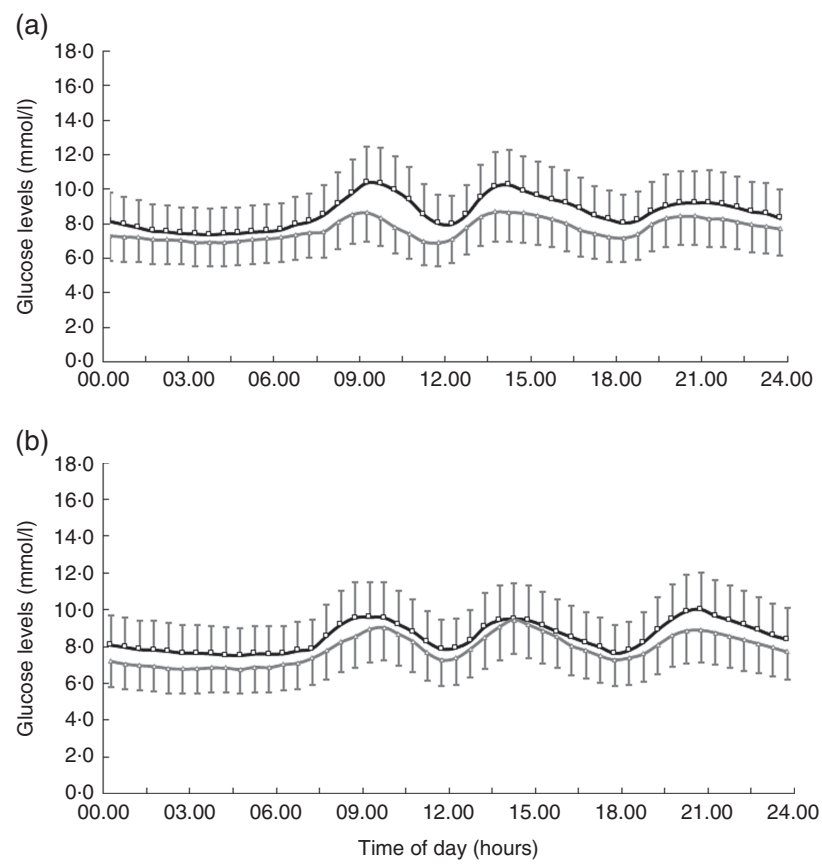

Fig. 2. Comparison of average continuous glucose monitoring tracings between the (a) lifestyle intervention ( $\mathrm{LI})+$ meal replacement (MR) group and the (b) LI group. The black curve represents the baseline values, and the grey curve represents the endpoint values. Data are reported as means and standard deviations.

excursions, it is possible that the effect of MR on postprandial glucose contributes to improvements in SDBG, CV and MAGE. However, it is noteworthy that MR was only applied at 
Table 3. Multivariate regression analysis investigating the determinants of improvements in metrics of glycaemic variability ( $\beta$-Coefficients and $95 \%$ confidence intervals)

\begin{tabular}{|c|c|c|c|c|c|c|c|c|c|c|c|c|}
\hline \multirow[b]{3}{*}{ Independent variables } & \multicolumn{12}{|c|}{ Dependent variable } \\
\hline & \multicolumn{3}{|c|}{ AUCpp } & \multicolumn{3}{|c|}{ SD } & \multicolumn{3}{|c|}{ CV } & \multicolumn{3}{|c|}{ MAGE } \\
\hline & $\beta$ & $95 \% \mathrm{Cl}$ & $P^{\star}$ & $\beta$ & $95 \% \mathrm{Cl}$ & $P+$ & $\beta$ & $95 \% \mathrm{Cl}$ & $P \ddagger$ & $\beta$ & $95 \% \mathrm{Cl}$ & $P \S$ \\
\hline $\begin{array}{l}\mathrm{LI}+\mathrm{MR}(v . \mathrm{LI}) \\
\Delta \mathrm{I}_{30} / \Delta \mathrm{G}_{30}\end{array}$ & $\begin{array}{r}-220.66 \\
-12.47\end{array}$ & $\begin{array}{c}-310 \cdot 74,130.58 \\
-23 \cdot 83,1 \cdot 12\end{array}$ & $\begin{array}{r}<0.001 \\
0.032\end{array}$ & $\begin{array}{l}-0.32 \\
-0.04\end{array}$ & $\begin{array}{l}-0.54,0.1 \\
-0.07,0.01\end{array}$ & $\begin{array}{l}0.004 \\
0.005\end{array}$ & $\begin{array}{l}-3.77 \\
-0.4\end{array}$ & $\begin{array}{l}-6.36,1.19 \\
-0.72,0.08\end{array}$ & $\begin{array}{l}0.005 \\
0.016\end{array}$ & $\begin{array}{l}-0.66 \\
-0.1\end{array}$ & $\begin{array}{l}-1 \cdot 26,0.06 \\
-0.18,0.03\end{array}$ & $\begin{array}{l}0.032 \\
0.008\end{array}$ \\
\hline
\end{tabular}

AUCpp, AUC of postprandial blood glucose; MAGE, mean amplitude of glycaemic excursions; LI, lifestyle intervention; MR, meal replacement.

* $P$ was adjusted for baseline clinical variables (age, sex, BMI, fasting plasma glucose, 2-h plasma glucose, $\mathrm{HbA}_{1 \mathrm{c}}, \Delta \mathrm{l}_{120} / \Delta \mathrm{G}_{120}$ and Matsuda index) and baseline $\mathrm{AUCpp}$.

$\dagger P$ was adjusted for baseline clinical variables as mentioned above and baseline SD.

$\ddagger P$ was adjusted for baseline clinical variables as mentioned above and baseline $C V$.

$\S P$ was adjusted for baseline clinical variables as mentioned above and baseline MAGE.
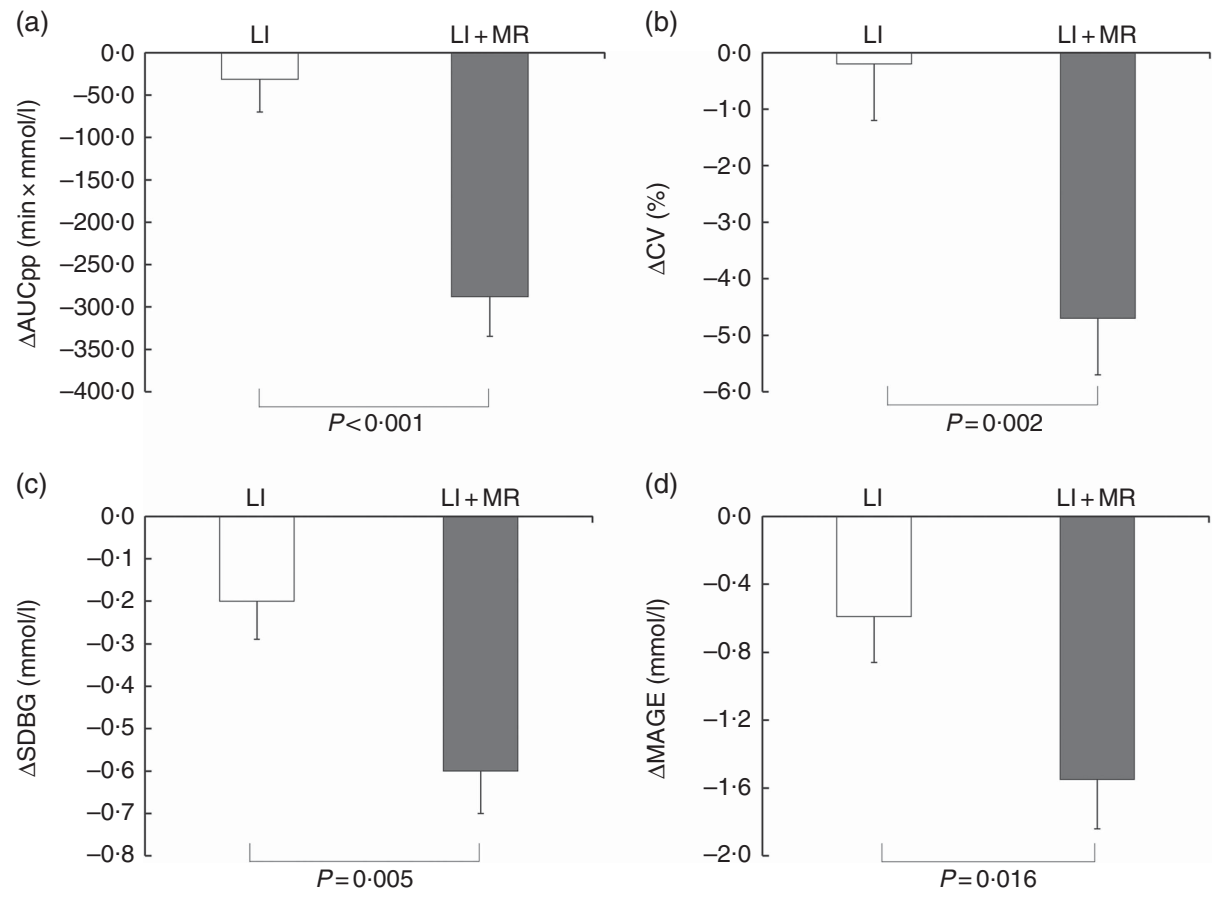

Fig. 3. Comparison of mean changes in (a) postprandial blood glycaemic excursions (incremental AUC of postprandial blood glucose (AUCpp)), (b) CV of glucose, (c) standard deviation of blood glucose (SDBG) and (d) mean amplitude of glycaemic excursions (MAGE) in the lifestyle intervention (LI) and LI+ meal replacement (MR) groups. Data are reported as means with their standard errors.

breakfast, and a previous study reported that breakfast MR did not affect postprandial glucose levels after lunch ${ }^{(9)}$. Therefore, other mechanisms may be involved in the promotion of GV by MR. There is emerging evidence that in addition to its physicochemical properties, dietary fibre also affects the composition of the gut microbiota, and the fermentation of dietary fibre in the intestine leads to increased production of SCFA, which stimulate the secretion of incretin hormones including glucagon-like peptide 1 (GLP-1) ${ }^{(30,31)}$. GLP-1 inhibits glucagon secretion and gastrointestinal motility, and acts as a physiological regulator of appetite. Moreover, it stimulates insulin secretion in a glucose-dependent manner ${ }^{(32)}$. Therefore, GLP-1 is regarded as a crucial player in the regulation of glucose homoeostasis. Interestingly, increases in GLP-1 during OGTT were reported to be significantly correlated with early phase insulin response $\left(\Delta \mathrm{I}_{30} / \Delta \mathrm{G}_{30}\right)^{(33)}$, which was consistently and independently associated with improvements in GV parameters in our study, suggesting that GLP-1 may, in part, account for the beneficial effect of MR on GV.

Glucerna SR is characterised by a higher MUFA:carbohydrate ratio, the presence of fructose and high amounts of fibre, which all lead to lower postprandial glucose levels. Of note, an indirect effect of MR on postprandial glucose in the real world may be of concern, although this could not be confirmed in the current study as the participants were instructed not to skip breakfast. In this regard, a clinical trial conducted by Jakubowicz et $a l .{ }^{(34)}$ showed that skipping breakfast resulted in a significantly higher glycaemic response after subsequent isoenergetic lunch and dinner accompanied by impaired responses of insulin and GLP-1. Moreover, they found that skipping breakfast acutely disrupts the circadian clock and the expression of clock-controlled genes, which are crucial for glucose metabolism $^{(35)}$. Considering that MR decreases the need for intensive dietary counselling, and is convenient and easy to 
prepare, especially for individuals staying away from home, it may possibly reduce the chance of breakfast skipping and therefore prevent postprandial glucose excursions.

As for other cardiometabolic risk factors, LI + MR demonstrated more pronounced improvements in systolic BP and TIR than LI. Two previous studies reported the beneficial effect of MR on BP as well. Chaiyasoot et $a l^{(2)}$ speculated that lower total energy intake caused by MR may partially account for this observation. However, the decrease in body weight did not differ significantly between the two intervention arms in our study, suggesting that MR may exert a weight-independent effect on BP. Although the two interventions led to similar improvements in $\mathrm{HbA}_{1 \mathrm{c}}$ and GA, significantly greater improvement in TIR was achieved with LI + MR than with LI. $\mathrm{HbA}_{1 \mathrm{c}}$ and GA reflect the average glucose level for a period of time and are widely used in clinical practice. However, it is recognised that they are far from perfect in the evaluation of glucose control. On the other hand, TIR refers to the time spent in the target glucose range and provides information about whether hypoglycaemia or hyperglycaemia improves over time, which is not reflected by $\mathrm{HbA}_{1 \mathrm{c}}$ or GA. Our findings further support the notion that TIR is a valuable metric for glucose control ${ }^{(36)}$ in addition to $\mathrm{HbA}_{1 \mathrm{c}}$ and GA.

Several limitations of the study design should be noted. First, the intervention period was relatively short (i.e. 4 weeks). Therefore, the long-term effects of MR on the cardiometabolic profile and GV could not be determined. Second, the openlabel design of the current study may introduce some extent of bias. However, since Glucerna SR looks and tastes different compared with a Chinese diet, a blinded trial was not feasible. Third, the energy content of the breakfast in the LI + MR groups were lower than those in the LI group, which may be a confounding factor for the relationship between MR and GV. However, since the weight loss was similar in the two groups and was only about $2 \%$, it is unlikely that the lower energy intake related with MR accounts for the improvements in GV. Finally, only untreated T2D patients were admitted in the study. Our findings may not be generalised to those patients receiving hypoglycaemic agents, and the possibility of evaluating the interaction between MR and different hypoglycaemic agents was precluded.

In conclusion, breakfast replacement with a liquid formula for 4 weeks significantly improved GV in T2D patients. Our results suggest that MR could be an effective treatment to alleviate glucose fluctuations, which is associated with the development of diabetic complications. Future studies with longer intervention times are warranted to confirm the beneficial effects of MR on GV.

\section{Acknowledgements}

The authors would like to thank all of the involved clinicians, nurses and technicians for dedicating their time and skill to the completion of this study.

This work was funded by the Shanghai Municipal Education Commission-Gaofeng Clinical Medicine Grant Support (20161430).
X. M. and J. Z. contributed to study design, data analysis and interpretation of data. J. P. and J. L. contributed to data analysis and writing the paper. L. Y., W. L. and W. Z. conducted the study and contributed to data collection. Y. B. interpreted the data and revised the manuscript. X. M. and J. Z. are the guarantors of this work and, as such, had full access to all the data in the study and take responsibility for the integrity of the data and the accuracy of the data analysis.

The authors declare that there are no conflicts of interest.

\section{References}

1. American Diabetes Association (2018) 4. Lifestyle management: Standards of Medical Care in Diabetes-2018. Diabetes Care 41, Suppl. 41, S38-S50.

2. Chaiyasoot K, Sarasak R, Pheungruang B, et al. (2018) Evaluation of a 12 -week lifestyle education intervention with or without partial meal replacement in Thai adults with obesity and metabolic syndrome: a randomised trial. Nutr Diabetes $\mathbf{8}, 23$.

3. Gulati S, Misra A, Tiwari R, et al. (2017) Effect of high-protein meal replacement on weight and cardiometabolic profile in overweight/obese Asian Indians in North India. Br J Nutr 117, $1531-1540$

4. Kempf K, Schloot NC, Gartner B, et al. (2014) Meal replacement reduces insulin requirement, HbA1c and weight longterm in type 2 diabetes patients with $>100 \mathrm{U}$ insulin per day. J Hum Nutr Diet 27, Suppl. 2, 21-27.

5. Leader NJ, Ryan L, Molyneaux L, et al. (2013) How best to use partial meal replacement in managing overweight or obese patients with poorly controlled type 2 diabetes. Obesity (Silver Spring) 21, 251-253.

6. Xu DF, Sun JQ, Chen M, et al. (2013) Effects of lifestyle intervention and meal replacement on glycaemic and bodyweight control in Chinese subjects with impaired glucose regulation: a 1-year randomised controlled trial. Br J Nutr 109, 487-492.

7. Gonzalez-Ortiz M, Martinez-Abundis E, Hernandez-Salazar E, et al. (2006) Effect of a nutritional liquid supplement designed for the patient with diabetes mellitus (Glucerna SR) on the postprandial glucose state, insulin secretion and insulin sensitivity in healthy subjects. Diabetes Obes Metab $\mathbf{8}$, 331-335.

8. Wang WQ, Zhang YF, Zhou DJ, et al. (2008) Open-label, randomized, multiple-center, parallel study comparing glycemic responses and safety profiles of Glucerna versus Fresubin in subjects of type 2 diabetes mellitus. Endocrine 33, $45-52$.

9. Stenvers DJ, Schouten LJ, Jurgens J, et al. (2014) Breakfast replacement with a low-glycaemic response liquid formula in patients with type 2 diabetes: a randomised clinical trial. $\mathrm{BrJ}$ Nutr 112, 504-512.

10. Monnier L, Colette C \& Owens D (2012) The glycemic triumvirate and diabetic complications: is the whole greater than the sum of its component parts? Diabetes Res Clin Pract 95 , 303-311.

11. Suh S \& Kim JH (2015) Glycemic variability: how do we measure it and why is it important? Diabetes Metab $J$ 39, 273-282.

12. Diabetes Control and Complications Trial Research Group, Nathan DM, Genuth S, et al. (1993) The effect of intensive treatment of diabetes on the development and progression of long-term complications in insulin-dependent diabetes mellitus. $N$ Engl J Med 329, 977-986. 
13. Holman RR, Paul SK, Bethel MA, et al. (2008) 10-year followup of intensive glucose control in type 2 diabetes. $N$ Engl J Med 359, 1577-1589.

14. Writing Group for the DERG, Orchard TJ, Nathan DM, et al. (2015) Association between 7 years of intensive treatment of type 1 diabetes and long-term mortality. JAMA 313, 45-53.

15. Picconi F, Parravano M, Ylli D, et al. (2017) Retinal neurodegeneration in patients with type 1 diabetes mellitus: the role of glycemic variability. Acta Diabetol 54, 489-497.

16. Soupal J, Skrha J Jr, Fajmon M, et al. (2014) Glycemic variability is higher in type 1 diabetes patients with microvascular complications irrespective of glycemic control. Diabetes Technol Ther 16, 198-203.

17. Matsutani D, Sakamoto M, Iuchi H, et al. (2018) Glycemic variability in continuous glucose monitoring is inversely associated with baroreflex sensitivity in type 2 diabetes: a preliminary report. Cardiovasc Diabetol 17, 36 .

18. Su G, Mi S, Tao H, et al. (2011) Association of glycemic variability and the presence and severity of coronary artery disease in patients with type 2 diabetes. Cardiovasc Diabetol 10, 19.

19. Horvath EM, Benko R, Kiss L, et al. (2009) Rapid 'glycaemic swings' induce nitrosative stress, activate poly(ADP-ribose) polymerase and impair endothelial function in a rat model of diabetes mellitus. Diabetologia 52, 952-961.

20. Quagliaro L, Piconi L, Assaloni R, et al. (2003) Intermittent high glucose enhances apoptosis related to oxidative stress in human umbilical vein endothelial cells: the role of protein kinase $\mathrm{C}$ and $\mathrm{NAD}(\mathrm{P}) \mathrm{H}$-oxidase activation. Diabetes $\mathbf{5 2}$, 2795-2804.

21. Monnier L, Mas E, Ginet C, et al. (2006) Activation of oxidative stress by acute glucose fluctuations compared with sustained chronic hyperglycemia in patients with type 2 diabetes. JAMA 295, 1681-1687.

22. Wentholt IM, Kulik W, Michels RP, et al. (2008) Glucose fluctuations and activation of oxidative stress in patients with type 1 diabetes. Diabetologia 51, 183-190.

23. Brownlee M (2005) The pathobiology of diabetic complications: a unifying mechanism. Diabetes 54, 1615-1625.

24. Lu J, Ma X, Zhou J, et al. (2018) Association of time in range, as assessed by continuous glucose monitoring, with diabetic retinopathy in type 2 diabetes. Diabetes Care 41, 2370-2376.

25. Matsuda M \& DeFronzo RA (1999) Insulin sensitivity indices obtained from oral glucose tolerance testing: comparison with the euglycemic insulin clamp. Diabetes Care 22, 1462-1470.

26. Cavalot F, Pagliarino A, Valle M, et al. (2011) Postprandial blood glucose predicts cardiovascular events and all-cause mortality in type 2 diabetes in a 14-year follow-up: lessons from the San Luigi Gonzaga Diabetes Study. Diabetes Care 34 , $2237-2243$

27. Coutinho M, Gerstein HC, Wang Y, et al. (1999) The relationship between glucose and incident cardiovascular events. A metaregression analysis of published data from 20 studies of 95,783 individuals followed for 12.4 years. Diabetes Care 22, 233-240.

28. Sartore G, Chilelli NC, Burlina S, et al. (2013) Association between glucose variability as assessed by continuous glucose monitoring (CGM) and diabetic retinopathy in type 1 and type 2 diabetes. Acta Diabetol 50, 437-442.

29. Wang X, Zhao X, Dorje T, et al. (2014) Glycemic variability predicts cardiovascular complications in acute myocardial infarction patients with type 2 diabetes mellitus. Int J Cardiol 172, 498-500

30. Zhou J, Martin RJ, Tulley RT, et al. (2008) Dietary resistant starch upregulates total GLP-1 and PYY in a sustained daylong manner through fermentation in rodents. Am J Physiol Endocrinol Metab 295, E1160-1166.

31. Zhao L, Zhang F, Ding X, et al. (2018) Gut bacteria selectively promoted by dietary fibers alleviate type 2 diabetes. Science 359, 1151-1156.

32. Holst JJ (2007) The physiology of glucagon-like peptide 1 . Physiol Rev 87, 1409-1439.

33. Shen J, Chen Z, Chen C, et al. (2013) Impact of incretin on early-phase insulin secretion and glucose excursion. Endocrine 44, 403-410.

34. Jakubowicz D, Wainstein J, Ahren B, et al. (2015) Fasting until noon triggers increased postprandial hyperglycemia and impaired insulin response after lunch and dinner in individuals with type 2 diabetes: a randomized clinical trial. Diabetes Care 38, 1820-1826.

35. Jakubowicz D, Wainstein J, Landau Z, et al. (2017) Influences of breakfast on clock gene expression and postprandial glycemia in healthy individuals and individuals with diabetes: a randomized clinical trial. Diabetes Care 40, 1573-1579.

36. Danne T, Nimri R, Battelino T, et al. (2017) International consensus on use of continuous glucose monitoring. Diabetes Care 40, 1631-1640. 